\title{
Treatment patterns, clinical outcomes, and healthcare resource use associated with advanced/metastatic lung cancer in China: protocol for a retrospective observational study
}

\author{
Bin Qiu ${ }^{1}$, Gaofeng $\mathrm{Li}^{2}$, Feng $\mathrm{Luo}^{3}$, Xiaohong $\mathrm{Cai}^{4}$, Lin $\mathrm{Wu}^{5}$, Jianhua Chen ${ }^{5}$, Yanping $\mathrm{Hu}^{6}$, Zhiliu Tang \\ Shuo Yang ${ }^{8}$, Jie $\mathrm{He}^{1}$ \\ ${ }^{1}$ Cancer Hospital, Chinese Academy of Medical Sciences, Beijing, China; ${ }^{2}$ Yunnan Cancer Hospital, Kunming, China; ${ }^{3}$ Lung Cancer Center, West \\ China Hospital, Sichuan University, Chengdu, China; ${ }^{4}$ Sichuan Cancer Hospital \& Institute, Chengdu, China; ${ }^{5}$ Hunan Cancer Hospital, Changsha, \\ China; ${ }^{6}$ Hubei Cancer Hospital, Hubei, China; ${ }^{7}$ Bristol-Myers Squibb, Shanghai, China (was with BMS at the time when the research and the \\ manuscript were conducted); ${ }^{8}$ Bristol-Myers Squibb, Shanghai, China \\ Correspondence to: Jie He, MD. Department of Thoracic Surgery, National Cancer Center, National Clinical Research Center for Cancer, Cancer \\ Hospital, Chinese Academy of Medical Sciences and Peking Union Medical College, 17 Panjiayuan Nanli, Chaoyang District, Beijing 100021, \\ China. Email: hejie@cicams.ac.cn.
}

Background Lung cancer (LC) is the most common cancer worldwide. The prevalence of LC and rate
of associated mortality are high and increasing faster in China than in Western countries. Non-small cell
lung cancer (NSCLC) accounts for most LCs. This study aims to be the first large, multi-center, non-
interventional retrospective study of treatment patterns (type/duration, number of lines, completion rate),
real-world outcomes, and medical costs among Chinese patients with advanced/metastatic NSCLC (IIIb/IV)
or extensive-stage small cell LC (ES-SCLC).
Methods: This study will enroll 8,800 patients ( $\geq 18$ years, with a diagnosis of advanced/metastatic NSCLC
made between 1 December 2013 to 30 November 2014 ) from 35 to 50 Chinese sites. Hospital information
systems (HIS) and electronic medical records will be retrospectively reviewed, in adherence with regulatory
and ethical requirements. Early-stage treatment (starting from 1 December 2010 ) of patients with recurrent
disease or early disease progression will be examined. Data will be collected at baseline (diagnosis) and 6 and
12 months after this. Observation will end after 3 years or death. Data will be stratified by histology, staging,
age, region, health insurance, and epidermal growth factor receptor (EGFR)/anaplastic lymphoma kinase
(ALK) mutation status. Treatment duration and overall survival will be estimated using Kaplan-Meier curves.
Descriptive statistics will be used for disease characteristics and patient demographics. Cox-proportional
hazards models will be used to examine the impact of demographics/treatment on survival. Treatment
patterns and outcome predictors will be explored using multivariate logistic regression.

Discussion: This protocol describes the methodology for collecting real-world data to guide evidencebased clinical practice and inform unmet needs in NSCLC treatment, with potential to identify gaps between guidelines and current practice.

Trial registration: NCT03505515; data registered on ClinicalTrials.gov: 12h Apr., 2018.

Keywords: China; lung neoplasms; clinical protocol

Submitted Oct 12, 2020. Accepted for publication Dec 18, 2020.

doi: $10.21037 /$ tlcr-20-1269

View this article at: http://dx.doi.org/10.21037/tlcr-20-1269 


\section{Introduction}

Lung cancer (LC) is the most common cancer worldwide. In China, LC claims more lives than any other malignancy, and its prevalence and mortality have rapidly increased in recent decades (1). The incidence of LC in China is high and the reported rate has risen faster in China than in Western countries $(1,2)$. Key risk factors that contribute to the disease burden of LC in China include smoking and air pollution (1). The National Central Cancer Registry estimated that 651,053 new patients were diagnosed with LC in 2011, representing $19.3 \%$ of all new cancer cases in China (2). The distribution of these newly diagnosed patients was similar between rural and urban areas (2). The incidence and mortality rates of LC are relatively low in individuals under 45 years of age, but increase with age (1). In China, LC has a higher prevalence in men than in women, with crude incidence rates of 61.9/100,000 and 29.5/100,000, respectively (1). A Shanghai study estimated that the crude incidences of non-small cell lung cancer (NSCLC) in men and women were 55.9/100,000 and $52.4 / 100,000$, respectively; this would suggest that NSCLC accounts for $\sim 90.3 \%$ of LCs reported in Chinese men (3). Historically (2011 and 2014 data), LC mortality rates have been higher in men than in women $(2,4)$.

Data from the US Surveillance, Epidemiology, and End Results (SEER) database (2014 data), show that 22\% of LC patients had advanced disease that had spread to regional lymph nodes, while $57 \%$ had metastasized cancer (5). Similarly, in China, most cases of LC are diagnosed at an advanced stage (6). A Chinese study of patients with NSCLC, indicated that stage IIIa and IIIb/IV accounted for $12.9 \%$ and $63.5 \%$ of patients, respectively, for whom the tumor stage was known (1,734/5,099 patients) (3). The majority of Chinese patients diagnosed with LC receive treatment according to the China Expert Consensus on the Diagnosis and Treatment of Advanced Stage Primary Lung Cancer (2016 version), a framework developed in parallel to international guidelines (7). The guidelines for the treatment of NSCLC recommend molecular pathology methods for the detection of epidermal growth factor receptor (EGFR) or anaplastic lymphoma kinase (ALK) gene mutations $(7,8)$. Systemic therapy, including molecular targeted treatment and chemotherapy, is the recommended first-line treatment for advanced NSCLC (8).

Small cell LC (SCLC), which accounts for approximately $15 \%$ of LCs in China (9). is a fast-developing, rapidly invasive cancer. At the time of diagnosis, $>70 \%$ of SCLC patients have metastasis, and the survival rate is low (9). Chemotherapy, including cisplatin-etoposide (EP), carboplatin-etoposide (EC), irinotecan-cisplatin (IP) and irinotecan-carboplatin IC, is the standard therapy for patients with extensive-stage (ES) SCLC (5).

Few large-scale national surveys have explored the treatment patterns, real-world outcomes, and direct economic burden of LC in China. A retrospective study using data from the Guangdong Lung Cancer Institute revealed treatment disparities among advanced NSCLC patients, most notably in the second-line setting (10). Shi et al. investigated SCLC treatment patterns in clinical practice across 12 medical centers, in 5 major Chinese cities (11). They concluded that the treatment options used in these hospitals were consistent with those of international recommendations (11). Site-based retrospective studies have examined real-world outcomes and the use of healthcare resource in China. In these studies, median overall survival (OS) was estimated to range from 13-17 months for patients with stage IIIb LC and from 8.4-24 months for patients with stage IV LC (12-17). Several studies that investigated the direct economic burden of LC in China report an estimated length of hospital stay in the range of 10.6-45.6 days, as well as inpatient expenses ranging from 8,816-58,512 RMB (\$1,280.3-8,495.5 USD), which accounted for 81.5-85.2\% of the direct economic burden (18-28).

Due to small sample sizes and a lack of geographical representation, current research in this area is limited in its application for evidence-based decision-making. This study aims to be the first large, multi-center, non-interventional retrospective study of treatment patterns, real-world outcomes, and direct medical costs among Chinese patients with advanced/metastatic NSCLC or ES-SCLC. This study protocol highlights the methodology to be used and the resulting study data will provide a baseline for comparison of treatments introduced since the 2017 data collection point.

\section{Methods}

\section{Design}

This study will enroll 8,800 patients from 35-50 sites in China. Inpatient and outpatient hospital information systems (HIS) and electronic medical records will be retrospectively reviewed, with a focus on patients diagnosed with advanced/metastatic NSCLC (IIIb/IV) or ES-SCLC between 1 December 2013 and 30 November 2014. The 
index date is the date of diagnosis of IIIb/IV NSCLC or ESSCLC, and each patient will be retrospectively observed for a period of 3 years from this date (from 1 December 2013 to 30 November 2017). For patients with recurrent disease or progression from early-stage disease, early-stage treatment patterns will be examined from 1 December 2010.

\section{Study objectives (data variables: Table 1)}

\section{Primary objectives}

(I) To ascertain the treatment patterns of subgroups of patients with advanced/metastatic LC [advanced NSCLC (IIIb/IV)] in China.

(II) To determine which patients receive systemic anticancer treatment (chemotherapy or biologic/targeted therapy), as well as the proportion of patients that receive second-, third-, and fourth-line systemic therapy.

(III) To describe the agents/regimens received, treatment duration, and completion rate for each line of therapy.

\section{Secondary objectives}

(I) To describe patient demographics and disease characteristics at baseline and at the beginning of each line of therapy, with stratification by histology, staging, age, geographic region, and type of health insurance.

(II) To describe OS by histology and line of therapy, the use of cancer-directed treatments (including surgery, radiation, and supportive care), the interval between each line of therapy, and LC-related healthcare costs.

\section{Exploratory objectives}

To explore the overall predictors of treatment patterns and outcomes of patients with advanced/metastatic LC and ES-SCLC, and early-stage treatment patterns prior to the enrolment period for patients with recurrent disease or progression from early-stage disease.

\section{Inclusion criteria}

Patients will be selected for inclusion based on the following criteria:

(I) Histology-confirmed advanced NSCLC (IIIb/IV) and ES-SCLC with pathology/cytology recorded between 1 December 2013 and 30 November 2014;

(II) Receiving treatment as described here as (i) a hospital inpatient, (ii) a hospital outpatient or (iii) an outpatient at a chemotherapy center: (a) Received systemic LC treatment as an inpatient, visiting a selected site more than twice (e.g., for diagnosis and treatment).

(b) Alternatively, for hospitals with outpatient records, patients who received oral tyrosine kinase inhibitor (TKI) therapy, and routinely followed up (more than twice a year) outpatients will be included.

(c) For hospitals with an outpatient chemotherapy center, patients will be included if they were prescribed chemotherapy and routinely (more than twice a year, inclusive) followed up in the hospital as an outpatient.

(III) $\geq 18$ years of age at initial diagnosis of IIIb/IV NSCLC and ES-SCLC.

\section{Exclusion criteria}

Patients will be excluded from the study if they meet any of the following criteria:

(I) Patients who have participated in or are attending clinical trials of LC therapies;

(II) Unknown initial diagnosis time or initial treatment time;

(III) For hospitals without an outpatient chemotherapy center, patients who were prescribed chemotherapy but have no inpatient records;

(IV) Patients who received therapy as an inpatient on one occasion but were not routinely followed up as an outpatient, having fewer than 2 visits to receive inpatient care per year.

\section{Data collection}

The selection of hospitals ( $35-50$ hospitals) will be geographically representative, by including sites located in developed and developing cities in different regions of China. Following feasibility assessment, sites will be selected from the National Centre of Cancer Registry Data will be sourced from inpatient and outpatient records, with the lead site being responsible for checking the quality of the data and ensuring completeness of outpatient and inpatient records from surgical and internal medicine oncology departments. Oral therapies, such as TKIs, may be prescribed in both inpatient and outpatient settings in China; usually intravenous chemotherapy is received by inpatients, but some large hospitals have outpatient chemotherapy centers to increase efficiency. Figure 1 illustrates the study timeline for patients with an initial 
Table 1 Outcome/endpoint variables

Patterns of chemotherapy and biologic therapy

- Treatment patterns will be analyzed based on histology and lines of treatment, as well as for predictive factors

- The frequency and duration of treatment will be determined for each line of treatment

- A list of the agents and regimens used will be compiled, with an investigation into the most common chemotherapy and biologic therapy regimens

Line of therapy

- First-line therapy: therapy received during the first 28 days after the initiation of treatment, with the number of cycles per regimen counted as intravenous (IV) administrations or prescription fills

- Maintenance therapy: therapy delivered after $\geq 4$ cycles of first-line treatment without disease progression and subsequent treatment being initiated within 6 weeks of first-line therapy

- Second-line therapy: therapy received after $\geq 4$ cycles of first-line treatment with a time gap of $>6$ weeks without chemotherapy/biologic treatment between 2 consecutive cycles of $>6$ weeks; or if after $<4$ weeks of first-line therapy, a new treatment (that was not included in the first-line regimen) was administered, regardless of the length of time since the end of the first-line therapy. If one product from a combination regimen was discontinued, this did not constitute a change in the line of therapy

- Third-line therapy: a new line of therapy received after $\geq 4$ cycles of second-line treatment with a time gap of $>6$ weeks without chemotherapy/biologic treatment; or if after $<4$ cycles of second-line therapy, a new treatment (that was not included in the second-line regimen) was administered regardless of the length of time since the end of the second-line therapy. If one product from a combination regimen was discontinued, this did not constitute a change in the line of therapy

- Fourth-line and beyond: A gap of > 6 weeks in third-line therapy, or evidence of the administration of systemic therapy (excluding any agents used in the third-line regimen), regardless of the length of time since the end of the third-line therapy.

Duration of systemic therapy

- Determined by calculating the difference between the initial and last date at which the first drug of a regimen was administered

Treatment modification

- Defined as; dose reductions, treatment interruptions (temporary pause in treatment with intent to resume), and treatment discontinuation. Where available, the reason for the modification will be noted

Overall survival

- Interval between the date of diagnosis and the date of death (stratified by histology at diagnosis and from time of initiation of systemic therapy)

- Deaths will be verified using death certificate searches at sites, reports from caregivers, relatives, or other healthcare providers

- The in-hospital mortality rate will be calculated first, followed by analysis of overall survival

- Analyzed for each line of therapy and key treatment regimens

- Use of other cancer-directed therapies

- Surgery

- Radiation therapy

- Traditional Chinese medicine

Use of BSC and palliative care

- Best supportive and palliative care are intended to alleviate pain, relieve symptoms, improve quality of life, and enhance the compliance of anti-cancer treatment

Ancillary procedures

- The frequency of ancillary procedures, such as biopsies and biomarker tests, will be documented and analyzed, overall and for each line of therapy

Total direct health care costs

- Post-index direct health care costs will be calculated for each patient, overall, and for each line of therapy

- Total direct cancer-related costs will be stratified by inpatient and outpatient status 


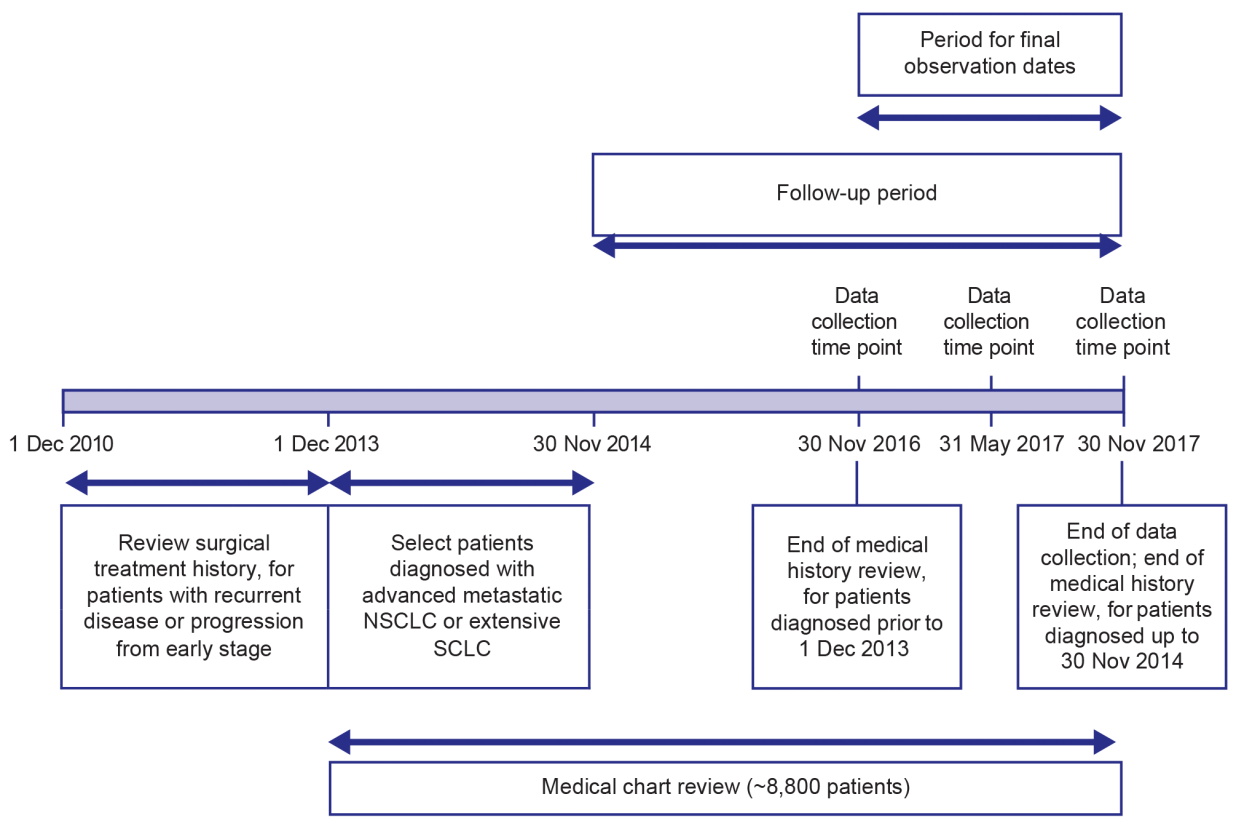

Figure 1 Study timeline for patients with an initial diagnosis of late-stage cancer.

diagnosis of late-stage cancer.

For patients with recurrent or progressive late-stage disease, the study will review the surgical treatment history from 1 December 2010 to the time of late-stage diagnosis (Figure 1; guidelines recommend surgery for patients with advanced NSCLC who show a good response to targeted therapy or chemotherapy as well as for patients with a single metastatic site) (8). As the sites of internal oncological care and where surgery is performed may differ, treatment histories for multiple sites may be obtained. Data will be collected at three specific time points: at baseline (diagnosis), and at 6 months and 1 year after diagnosis/initiation. Figure 1 shows the timeline for data collection. Depending on the time of diagnosis, the initiation, observational, and data collection periods may differ among patients. Observation will end 3 years after study initiation or on the death of the patient.

\section{Data source and management}

The China HIS will serve as a data source. HIS is an information management system that is widely used in Chinese hospitals, which was designed to manage administrative and clinical functions. Eligible patients will be identified using HIS, electronic medical records (EMRs), and/or chart review. Figure 2 illustrates the patient screening and data extraction process.

\section{Study variables}

Primary outcomes and endpoints of interest include: patterns of chemotherapy and biologic therapy use; OS; the use of other cancer-directed therapies, best supportive care (BSC) and palliative care; ancillary procedures; and direct healthcare costs. The patient outcomes and endpoints will be assessed at initial diagnosis and during the postdiagnostic follow-up (including during treatment with firstline-, maintenance-, and all subsequent lines of -therapy, where applicable). Patients will be censored at the end of a data stream. Further details on these variables are shown in Table 1, with additional information detailed in Table S1.

Independent variables of interest include patient demographics and risk factors, disease profile (including time of diagnosis, histology, staging, and mutation status), admission/discharge status, information relating to the hospital type and size, and the treating department. These data will be collected where available, and any missing data and the resulting potential impact will be documented.

\section{Statistical analysis}

Statistical analyses will be performed using SAS version 9.3 statistical software (SAS Institute Inc., Cary, North 


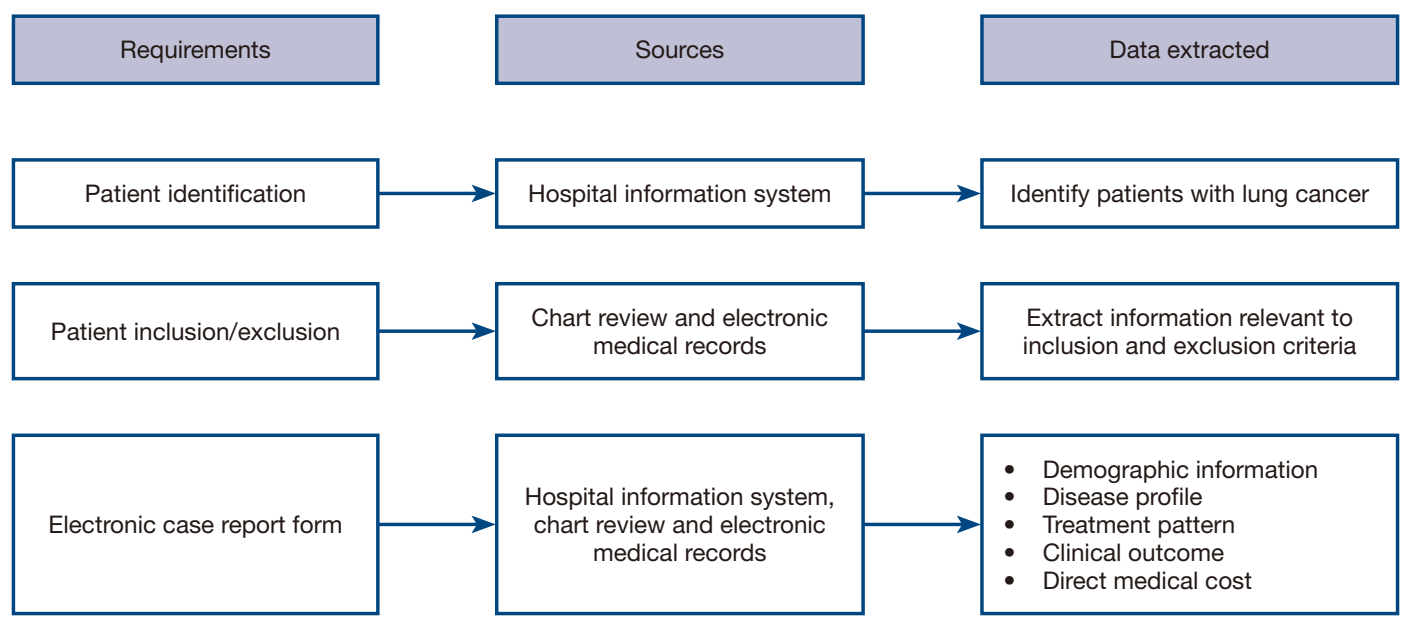

Figure 2 Depiction of the patient screening and data extraction process.

Carolina, USA) with an a priori significance level set at 0.05 . For primary objectives, treatment patterns will be analyzed according to histology, stage, line of therapy (first-line, maintenance, second-line, and beyond), age, region, and type of health insurance. Categorical variables will be presented as percentages, while continuous variables will be reported as mean, standard deviation (SD), median, and interquartile ranges. To analyze the treatment duration of each line of therapy, Kaplan-Meier (KM) curves will be estimated, using the date of the first dose administered as the initiation date and the date of the last infusion or projected final oral dose as the end date (whichever comes first).

For secondary objectives, patient demographics, disease characteristics, adjusted and unadjusted hospital charges, and other cancer-directed therapies will be presented using descriptive statistics. KM survival curves will be used to estimate survival probability for each patient, and Coxproportional hazards models will be used to examine whether differences in patient OS between regimens or treatments can be accounted for by controlling baseline demographics and clinical variables.

Exploratory objectives will be analyzed using multivariate logistic regression models to identify which observed patient and clinical factors are predictive of specific events associated with treatment patterns. Separate models will be used to analyze impacts of chemotherapy on treatment pattern associated events (versus any other cancer-directed treatment, among patients who received cancer-directed treatment). Multivariate generalized linear models (GLMs) will be generated to assess cost drivers for patients who received some form of cancer-directed therapy.
Mean and $95 \%$ confidence intervals (CIs) for proportions of patients receiving a particular treatment pattern (e.g., second-, third- and fourth-line) will be calculated for a sample size of 8,800 patients. In a descriptive setting, the sample size is related to the level of precision in estimating the primary outcomes. For this study, the intended analyses for the primary objectives are only descriptive; therefore, the precision of any estimates (i.e., the size of the CIs) will be affected by the sample size. Several descriptive objectives explore the treatment pattern of second-line therapy. To ensure sufficient power for the smallest histologic group, the proportion of patients receiving a certain regimen in second-line SCLC therapy will be used to calculate the subgroup sample size and back-calculate the total sample size based on the subgroup percentage.

\section{Ethics approval}

This observational study will be conducted in accordance with International Society for Pharmacoepidemiology (ISPE) Guidelines for Good Pharmacoepidemiology Practices and the applicable regulatory requirements. Prior to the initiation of the study, Ethics Committee/ Institutional review board approval will be obtained, as will the informed consent of patients, in compliance with the relevant laws and regulations.

\section{Discussion}

In Western countries, the incidence of LC is declining; in contrast, over the past few decades, China has experienced 
an increase in the incidence of LC, which has been attributed to tobacco use and air pollution (29). Consistent with Western countries, adenocarcinoma has replaced squamous cell carcinoma as the predominant type of LC in China (29-32). The incidence of EGFR mutations in Chinese LC patients is in the range of $40.3-64.5 \%$ for those with adenocarcinoma and $28.4 \%$ for NSCLC, which is far higher than the incidence of $3.0-42.0 \%$ seen in European and North American patients with NSCLC $(29,33)$. Current treatment practices focus on recommendations from the China Expert Consensus on the Diagnosis and Treatment of Advanced Stage Primary Lung Cancer (2016 version) (8); however, there is still a need for reports on real-world outcomes that can guide evidence-based clinical practice and provide information on unmet clinical needs. These real-world data have the potential to contribute towards a strategy that will improve the quality of care for patients with LC in China.

Although some previous studies have focused on LC treatment patterns, real-world outcomes, and health care utilization in China, none of the literature, to date, has successfully captured all parameters on a large scale, in a geographically representative manner (11,18-28). With the aim of addressing this knowledge gap, this study is appropriately powered according to the descriptive objectives and the optimal sample size determined. It has been designed to include data collection from a wide range of geographical sites, ensuring the inclusion of undeveloped and developed areas. The selection of a geographically representative cohort of patients and hospitals will minimize the potential selection bias, and by its design, this protocol is likely to capture a relatively complete snapshot of treatment patterns and outcomes among LC patients in China.

This study will reflect real-world treatment patterns and outcomes and, importantly, the direct medical costs that potentially have a substantial impact on the national healthcare system. Given the retrospective nature of the study and the difficulty in collecting comparable accurate and meaningful safety data for different treatments, comparisons of drug safety will not be made in this study. It is expected that this study will provide a deeper understanding of any unmet medical needs, through its identification of potential treatment gaps between current practice and guideline recommendations. This may allow areas for improvement to be identified in clinical practice and resource allocation for LC treatment. Furthermore, the robust, large-scale methodology of this protocol will enable the analysis and evaluation of pre-immuno-oncology
(IO) treatment patterns and the real-world implications in NSCLC and SCLC. By doing this, we anticipate that this study will set a benchmark for future studies and could even pave the way for post-IO comparisons in China.

This study is not without limitations. The use of HIS as a primary data source limits access to additional information such as socioeconomic status, health behaviors, and qualityof-life. Differences in inter- and intra-hospital practices may contribute to variations in data, and in large hospitals, where the enrolment population may contain a higher proportion of patients with severe disease, there is also potential for OS bias.

\section{Conclusions}

In conclusion, here, we have presented the protocol for the first large-scale, geographically representative study to examine the treatment patterns, real-world outcomes, and direct medical costs of patients diagnosed with advanced/ metastatic NSCLC or ES-SCLC in China.

\section{Acknowledgments}

Funding: The study was funded by Bristol-Myers Squibb Pharmaceuticals Ltd. Editorial assistance in manuscript preparation was provided by Anita Abeygooneseskera and Rachael Profit of MediTech Media, Asia Pacific, and funded by Bristol-Myers Squibb Pharmaceuticals Ltd., China.

\section{Footnote}

Conflicts of Interest: All authors have completed the ICMJE uniform disclosure form (available at http://dx.doi. org/10.21037/tlcr-20-1269). ZT was employed by BristolMyers Squibb at the time the manuscript was prepared, and SY is employed by Bristol-Myers Squibb. The other authors have no conflicts of interest to declare.

Ethical Statement: The authors are accountable for all aspects of the work in ensuring that questions related to the accuracy or integrity of any part of the work are appropriately investigated and resolved. This observational study will be conducted in accordance with International Society for Pharmacoepidemiology (ISPE) Guidelines for Good Pharmacoepidemiology Practices and the applicable regulatory requirements. Prior to the initiation of the study, Ethics Committee/Institutional review board approval will be obtained, as will the informed consent of patients, in 
compliance with the relevant laws and regulations.

Open Access Statement: This is an Open Access article distributed in accordance with the Creative Commons Attribution-NonCommercial-NoDerivs 4.0 International License (CC BY-NC-ND 4.0), which permits the noncommercial replication and distribution of the article with the strict proviso that no changes or edits are made and the original work is properly cited (including links to both the formal publication through the relevant DOI and the license). See: https://creativecommons.org/licenses/by-nc-nd/4.0/.

\section{References}

1. Chen W, Zheng R, Zeng H, et al. Epidemiology of lung cancer in China. Thorac Cancer 2015;6:209-15.

2. Zheng R, Zeng H, Zuo T, et al. Lung cancer incidence and mortality in China, 2011. Thorac Cancer 2016;7:94-9.

3. Fan H, Shao ZY, Xiao YY, et al. Incidence and survival of non-small cell lung cancer in Shanghai: a population-based cohort study. BMJ Open 2015;5:e009419.

4. Zheng XQ, Huang JF, Lin JL, et al. Incidence, prognostic factors, and a nomogram of lung cancer with bone metastasis at initial diagnosis: a population-based study. Transl Lung Cancer Res 2019;8:367-79.

5. Institute NC. Surveillance, Epidemiology, and End Results (SEER) Program. 2014. Available online: https://seer. cancer.gov/statfacts/html/lungb.html. Accessed 14 January 20202020

6. Ulrich BC, Guibert N. Immunotherapy efficacy and gender: discovery in precision medicine. Transl Lung Cancer Res 2018;7:S211-3.

7. Ettinger DS, Aisner DL, Wood DE, et al. NCCN Guidelines Insights: Non-Small Cell Lung Cancer, Version 5.2018. J Natl Compr Canc Netw 2018;16:807-21.

8. Shi Y, Sun Y, Yu J, et al. China Experts Consensus on the Diagnosis and Treatment of Advanced Stage Primary Lung Cancer (2016 Version). Zhongguo Fei Ai Za Zhi 2016;19:1-15.

9. Liu J, Cheng Y, Li H, et al. Current status of small cell lung cancer in China. Journal of Cancer Biology \& Research 2014;2:1032-36.

10. Yang LL, Zhang XC, Yang XN, et al. Lung cancer treatment disparities in China: a question in need of an answer. Oncologist 2014;19:1084-90.

11. Shi Y, Xing P, Fan Y, et al. Current small cell lung cancer treatment in China. Thorac Cancer 2015;6:233-8.

12. Wu M, Wang Y, An T, et al. Analysis of prognostic factors in 541 female patients with advanced non-small cell lung cancer. Zhongguo Fei Ai Za Zhi 2011;14:245-50.

13. Zhang C, Wang L, Li W, et al. Surgical outcomes of stage IV non-small cell lung cancer: a single-center experience. J Thorac Dis 2019;11:5463-73.

14. Peng H, Han HB, Li XQ et al. Analysis of clinical characteristics and survival of 1279 patients with lung cancer. Chinese Journal of Lung Cancer 2011;21:354-8.

15. Wang L, He Z, Yang S, et al. The impact of previous therapy strategy on the efficiency of anlotinib hydrochloride as a third-line treatment on patients with advanced non-small cell lung cancer (NSCLC): a subgroup analysis of ALTER0303 trial. Transl Lung Cancer Res 2019;8:575-83.

16. Zhou T, Wu C, Zhang C, et al. A retrospective study of lowdose apatinib combined with S-1 in patients with advanced non-small cell lung cancer. J Thorac Dis 2019;11:1831-7.

17. Dong K, Liang W, Zhao S, et al. EGFR-TKI plus brain radiotherapy versus EGFR-TKI alone in the management of EGFR-mutated NSCLC patients with brain metastases. Transl Lung Cancer Res 2019;8:268-79.

18. Gu YY. Epidemiology characteristics and disease burden of lung cancer patients with Shanghai identity [Dissertation]: Fu Dan University; 2013.

19. Fan XH, Feng Y, Shao W, et al. Application and outcome of clinical pathway in radio- and chemo-therapy in lung cancer patients. Chinese Hospital Management 2011;2:25-7.

20. Chen Z. Inpatient surgery cost structure and trend analysis of nearly 10,000 lung cancer patients. Chinese Health Research 2013;3:3.

21. Du $W$ Zhang $X$, Gao C, et al. New grey correlation analysis of hospitalization expenses of 600 patients with non-small cell lung cancer. Journal of Shanghai Jiaotong University (Medical Science) 2012;10:1356-9.

22. Gao YX, Xiao J, Wu XM, et al. Factor analysis of direct inpatient cost of 1666 lung cancer patients. Chinese Journal of Health Statistics 2011;3:278-80.

23. Ding TT, Zhang X, Gao C, et al. Impact analysis and cost control research on inpatient medical cost of patients with non-small cell lung cancer. Chinese Journal of Health Statistics 2012;2:240-2.

24. Gao YX, Yang M, Liu G, et al. Path analysis of impact factors of inpatient medical cost of patients with lung cancer. Chinese Journal of Public Health 2012:253-4.

25. Shang M. Economic burden and impact factors of inpatient lung cancer patients [Dissertation]: Shandong University 2013. 
26. Gao QQ, Li SX, Wang Y, et al. Impact analysis of inpatient cost in lung cancer patients who underwent surgery using Grey relational method. Health Economics Research 2013;12:56-8.

27. Lv HL, Zhao SF, Xie XP, et al. Impact analysis of inpatient cost of 16,866 lung cancer patients in Si Chuan Province. Chinese Journal of Evidence-Based Medicine 2013;11:1283-7.

28. Gong H, Liu Y, Ma L, et al. Inpatient cost analysis of patients with lung cancer and liver cancer from 1996 to 2006 in Lanzhou City. Modern Preventive Medicine 2012;39:270-2.

29. Zhou C. Lung cancer molecular epidemiology in China: recent trends. Transl Lung Cancer Res 2014;3:270-9.

Cite this article as: Qiu B, Li G, Luo F, Cai X, Wu L, Chen J, Hu Y, Tang Z, Yang S, He J. Treatment patterns, clinical outcomes, and healthcare resource use associated with advanced/metastatic lung cancer in China: protocol for a retrospective observational study. Transl Lung Cancer Res 2020;9(6):2460-2468. doi: 10.21037/tlcr-20-1269
30. Toyoda Y, Nakayama T, Ioka A, et al. Trends in lung cancer incidence by histological type in Osaka, Japan. Jpn J Clin Oncol 2008;38:534-9.

31. Thun MJ, Lally CA, Flannery JT, et al. Cigarette smoking and changes in the histopathology of lung cancer. J Natl Cancer Inst 1997;89:1580-6.

32. Devesa SS, Bray F, Vizcaino AP, et al. International lung cancer trends by histologic type: male:female differences diminishing and adenocarcinoma rates rising. Int $\mathrm{J}$ Cancer 2005;117:294-9.

33. Midha A, Dearden S, McCormack R. EGFR mutation incidence in non-small-cell lung cancer of adenocarcinoma histology: a systematic review and global map by ethnicity (mutMapII). Am J Cancer Res 2015;5:2892-911. 


\section{Supplementary}

Table S1 Definitions of lines of therapy

Initial lines of therapy

Each line of therapy is defined by the temporal relationship and sequencing of treatment received

First line

- A treatment is considered first-line when the patient receives chemotherapy, radiation therapy, and/or biologic medication during the first 28 days after the initiation of treatment

- The number of cycles per regimen will be determined from the number of IV administrations and oral prescription fills

- The regimen is only considered complete if the number of cycles is $>4$

Maintenance

- A treatment is considered maintenance therapy when the patient has received $\geq 4$ cycles of first-line therapy without disease progression

- The key clinical distinction between maintenance and second-line therapy is the presence of disease progression in the latter.

- Where clinical notes document disease progression, this will be used to indicate a change to second-line treatment. However, in the event of information regarding disease progression not being documented and $\geq 4$ cycles of first-line therapy having been completed:

- If subsequent treatment is initiated within 6 weeks, this will be classified as maintenance therapy

- If there is a gap of $>6$ weeks before subsequent treatment, this will be classified as second-line therapy

Subclassification of maintenance therapy:

- Continuation: defined as the continuation of $\geq 1$ agent from the first-line setting after 4 to 6 cycles, within 6 weeks of completion of the initial regimen, without disease progression

- Switching: defined as the continuation of agents different to those received in the first-line setting after 4 to 6 cycles of first-line therapy, within 6 weeks of completion of the initial regimen, without disease progression.

Subsequent lines of therapy

For patients who received first-line therapy, consecutive treatment cycles will be compared to examine any changes in therapy

Second-line

- A treatment is considered second line when a patient receives a new therapy after they have completed $\geq 4$ cycles or first-line therapy, with a time gap of $>6$ weeks without chemotherapy and/or biologic therapy between 2 consecutive cycles; or

- A patient has completed $<4$ cycles of first-line therapy and there is evidence of subsequent administration of a new treatment regimen not including any agent from the first-line regimen, regardless of the duration of time since the end of the first-line therapy

Note, the discontinuation of a single drug from a combination regimen will not be considered a change in line of therapy

Third-line

- A treatment is considered third-line when a patient receives a new line of therapy after completing $\geq 4$ cycles of second-line therapy, with a time gap of $>6$ weeks without chemotherapy and/or biologic treatment between 2 consecutive cycles, or

- If after $<4$ cycles of second-line therapy, a new treatment (that was not included in the second-line regimen) was administered regardless of the length of time since the end of the second-line therapy

Note, discontinuation of a single drug from a combination regimen will not be considered a change in line of therapy

Fourth-line and beyond

- A treatment is considered fourth-line when there is a gap of $>6$ weeks in third-line therapy, or

- There is evidence of the administration of systemic therapy (excluding any agents used in the third-line regimen), regardless of the length of time since the end of the third-line therapy 